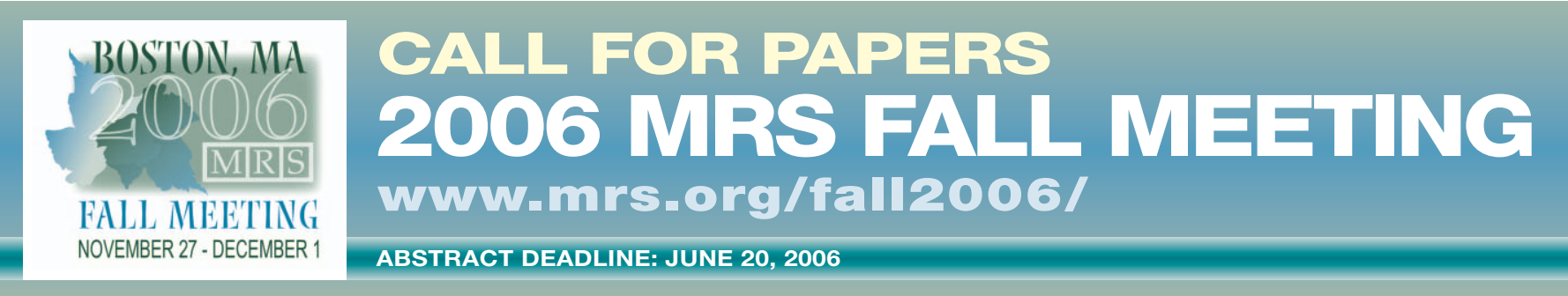

\title{
POSTERMINARIES
}

My favorite domestic product is undoubtedly bleach. Is your coffee mug looking stained? Simple washing doesn't clean it? Just add a dash of bleach, leave for a few minutes, and rinse. Is the shower cubicle beginning to collect those inevitable black lines of mold? Brush bleach on, walk away, run the shower again later, and it's all gone. I could go on, but I won't, because this is not a commercial for household products-it's about recycling.

In many countries, it is now the practice to separate our trash, most of which is packaging, into four or five different containers. In my household, we sort into glass, paper, plastics and metal, garden waste, and "the rest." Modern standards for house design are beginning to specify that every new dwelling should have space for at least five trash containers. This would entail a complete redesign of our apartment, in which the kitchen is already festooned with plastic bags containing other plastic bags, and rows of bottles waiting to be carried individually to their public collection point.

Although recycling has caught the public imagination as a self-evidently good thing, has anyone given enough consideration to the difficult issues it raises in a thoughtful household? How much time is being wasted while we, perplexed, stand in front of the five containers holding a piece of packaging in which plastic and paper are permanently welded together. Where to put it? What about those wonderful confectionary wrappers which are metallic on one side and paper on the other? My father once bet me five bob that I could not separate the two layers. It took me half an hour, but I did itperhaps that's the point at which I committed to becoming an engineer. Five bob, by the way, was five English shillings, now known as 25p, or less than $50 \notin$. In the 1960s, that would be enough to buy a new piece of rolling stock for my model railway (another marker on the way to geekdom and engineering).

\section{A Brown Study}

A further dilemma is whether to wash the items to be thrown away. Which is the minimum-energy way forward? What is the cost of the mental anguish of a tidy person contemplating putting a plastic container in the proper bin while it still contains substantial traces of coleslaw?

Although recycling has caught
the public imagination as a self-
evidently good thing, has anyone
given enough consideration to
the difficult issues it raises in
a thoughtful household?

Bottles represent further anguish. Until recently, we were encouraged to separately file these as clear, green, or brown, resulting in hesitation-potentially extending to tension headaches-over the very pale-tinted ones, or those blue overpriced water bottles. More recently, a touch of intellectual honesty has emerged, recognizing the fact that it is virtually impossible to imagine that color purity could be maintained in public bottle banks. Last week, our three neighborhood bottle banks were all over-painted with the slogan "use for all colors of glass."

Children soon learn, while painting, that mixing colors always leads, eventually, to brown. The same must be true, surely, for recycled materials. So what happens to the mixed glass, or the plastic bottles with contrastingly colored caps? For the glass, I suspect that it does not matter much, because its recycled use is likely to be undramatic and hidden-as road fill, for instance. But recycled plastics have a great future. The mundane reality in Europe is that we use plastic bags for our groceries and that much of this material is collected, sent to China, and returned to Europe as more (basically white) plastic bags. This seems a huge waste of energy, but I suppose that you can load an awful lot of plastic bags onto one ship! However, the more imagi- native thing to do is to exploit the inevitable tendency to converge on a muddy color and to recycle plastics as products which we want to be brown. As it happens, on both sides of the Atlantic, there is a need for vast volumes of brown products for use as fencing and decking. What could be better? We can exploit the intrinsic nondegradability of most polymers by using them for purposes where we want them to last forever in a hostile, wet environment. And at the same time, we can save trees! I am told that the volume of solid brown stuff needed just for fencing exceeds by two or three orders of magnitude the volume of synthetic polymers produced each year. Here, on our doorstep, is a real use for waste plastics. No worries about purity, color, or shipping around the world-just melt and extrude, or hot-compress, and you have a fence post, decking plank, railway sleeper, building block, or roadside curb.

A couple of interesting points come out of this analysis. The lifetime of the secondary recycled product is likely to be orders of magnitude longer than that of the primary product, which gives a better perspective on the life-cycle analysis of apparently short-lived products such as plastic milk bottles. An even more significant consequence is the drive to produce more polymeric materials. At current rates of production, we just don't have enough scrap plastic to fulfill society's need for fence posts. It will become a civic duty to consume plastic bags and bottles so that our state parks can have the decking and fencing they deserve without the loss of a single tree!

But what if you do want clear glass, or white plastic, from your recycled materials? If you want to be able to tell Chartreuse from Grand Marnier without opening the bottle, or to see out of the window? Or don't like to think of milk coming in a brown bottle? Then you have to give thanks for the industrial equivalent of my household bleach.

Peter GOODHEW 